\title{
Preserved visual representations despite change blindness in infants
}

\author{
Su-hua Wang ${ }^{1}$ and Stephen R. Mitroff ${ }^{2}$ \\ ${ }^{1}$ Department of Psychology, University of California, Santa Cruz, USA \\ 2 Department of Psychology and Neuroscience, Duke University, Durham, USA
}

\begin{abstract}
Combining theoretical hypotheses of infant cognition and adult perception, we present evidence that infants can maintain visual representations despite their failure to detect a change. Infants under 12 months typically fail to notice a change to an object's height in a covering event. The present experiments demonstrated that 11-month-old infants can nevertheless maintain a viable representation of both the pre- and post-change heights despite their 'change blindness'. These results suggest that infants, like adults, can simultaneously maintain multiple representations, even if they do not optimally use them.
\end{abstract}

\section{Introduction}

One of the central issues for developmental researchers is to unveil the nature of visual representations. How much information do young children represent visually and how do such representational abilities develop with experience? One especially useful method for investigating visual representations in infants (e.g. LeCompte \& Gratch, 1972; Muller \& Aslin, 1978) and adults (e.g. Simons \& Rensink, 2005) is the change-detection task, wherein a display is altered during a visual disruption and the primary question is whether or not the change is noticed. For example, infants watch a physical event in which an object array becomes fully hidden behind a screen, in sand, or under a cover. After a pause, the same (no-change event) or a different (change event) array is revealed. Results demonstrating that infants look reliably longer at the change than at the no-change event are taken to indicate that infants (1) represent relevant information of the first array, (2) maintain that representation through the disruption, (3) compare it with the second array, and (4) notice the difference in the change event. Using this method, recent research has revealed exciting new findings (e.g. Baillargeon, Li, Ng \& Yuan, in press; Cheries, Wynn \& Scholl, 2006; Káldy \& Leslie, 2005; Newcombe, Huttenlocher \& Learmonth, 1999; Wilcox, 1999), one of which concerns event-category effects - although infants have no difficulty in detecting an impossible change that occurs in events from one category, they may fail to detect the same change when it occurs in events from another category (e.g. Wang \& Baillargeon, 2006; Wilcox \& Chapa, 2002).

For example, Wang \& Baillargeon (2006) showed 11-month-old infants an event in which a rigid, inverted container (i.e. a cover) hid an object. At the beginning of the event, the object stood next to the cover. The cover was lifted and then lowered either in front of (occlusion condition) or directly on top of (covering condition) the object, fully hiding it. Note that the only difference between the two conditions was the relative depth at which the cover was

Address for correspondence: Su-hua Wang, Department of Psychology, University of California, 1156 High Street, Santa Cruz, CA 95064, USA; e-mail: suhua@ucsc.edu. 
lowered. In both conditions, when the cover was lifted again, the object was either the same as (no-change event) or much taller than (change event) before. The infants in the occlusion condition looked reliably longer at the change than at the no-change event, whereas those in the covering condition looked about equally at the two events, suggesting that, even though the infants had no difficulty detecting the change to the object's height in the occlusion event, they appeared blind to the same change in the covering event. ${ }^{1}$ Such event-category effects have been obtained previously with various age groups and event categories (Wang, 2008; Wang \& Baillargeon, 2006).2

The systematic pattern of infants' success and failure to detect impossible changes suggests a theoretical account of visual representation and object processing (Wang \& Baillargeon, 2008). The account proposes that three computational systems are involved when infants represent a simple physical event. An object-tracking system assigns an index to each object; each index 'sticks' to its object and helps infants track it as it moves during the event (e.g. Pylyshyn, 2001; Scholl \& Leslie, 1999). An object-representation system builds a detailed representation of each object, listing various object properties (e.g. Needham, 2001); each object representation is bound to its index so that infants can keep track of the properties of each object (e.g. Feigenson, Carey \& Hauser, 2002; Leslie, Xu, Tremoulet \& Scholl, 1998). As objects begin to move and interact, a physical-reasoning system categorizes the event based on spatiotemporal cues (e.g. Casasola, 2005; Rigney \& Wang, 2008). The physical-reasoning system then taps infants' knowledge about the selected category; the knowledge specifies what variables are relevant to predicting the outcomes of events from the category (e.g. Luo \& Baillargeon, 2005; Wang \& Kohne, 2007). According to this knowledge, the physicalreasoning system gathers information about the specified variables, either directly from the event if the objects are still visible or by querying the object-representation system if not. In each case, variable information is included in the representation of the event and interpreted in accord with physical rules that infants have acquired (e.g. Baillargeon, 2008; Leslie, 1994; Spelke, 1994). An important point to note here is that variable information not used by the physical-reasoning system may still be available in the object-representation system. When facing a task that taps only the object-representation and not the physical-reasoning system for example, a recognition-memory task that uses static displays and involves no object movement or interaction - infants might become able to retrieve and use the information. The present research tested this hypothesis.

Research on visual cognition with adults has shown that failures to detect changes can occur for a variety of reasons (e.g. Beck \& Levin, 2003; Hollingworth, 2003; O'Regan \& Noë, 2001; Pashler, 1988; Simons \& Rensink, 2005). In line with the above hypothesis, a recent study provided evidence for change blindness resulting from a failure to use available information (Mitroff, Simons \& Levin, 2004). College students briefly viewed an array of linedrawn objects. The array disappeared and then reappeared with the possibility that one of the objects was replaced with a new item. The participants reported whether they noticed the change and completed two-alternative forced-choice questions about which objects they believed had been in the display. On every trial, one question pitted the pre-change object against a novel object, and a second question pitted the post-change object against a novel

\footnotetext{
${ }^{1}$ Because the occlusion and covering conditions were identical except for the manner in which the object was hidden, the infants' differing responses suggest that they were attending to the majority of the event and not just representing the start and end states.

${ }^{2}$ Converging evidence for event-category effects is also obtained by violation-of-expectation tasks wherein the outcome of an interaction between objects violates a physical law. For example, an interaction violation occurs when an object is much shorter than and yet fully hides another object. Research showed that 3.5-month-old infants succeed in detecting such a violation when a tall object becomes fully hidden behind a short object; however, it is not until they are about 12 months of age that infants begin to detect the same violation when the tall object becomes fully hidden under the short object (e.g. Hespos \& Baillargeon, 2001, 2006; Wang, Baillargeon \& Paterson, 2005). In line with the findings in Wang \& Baillargeon (2006), 11-month-olds detect the above interaction violation when it occurs in occlusion but not in covering events.
} 
object. When the participants reported not noticing the change, they were still able to choose both the pre- and post-change objects at above-chance levels, suggesting that change blindness can occur from a failure to compare preserved representations and does not arise solely from failures to form and maintain viable representations. It is worth noting that, although the preand post-change objects were both selected at above-chance levels, the post-change objects were chosen at a much higher level of accuracy, indicating that the representations of the postchange objects were maintained more robustly than those of the pre-change objects.

The present research integrates the theoretical hypotheses of Wang \& Baillargeon (2008) and Mitroff et al. (2004a), and examines whether relevant information is still available even if infants fail to notice a change in an event. Might we obtain a dissociation in infants' response, as in adults', such that when they miss a change, they still have both the pre- and the postchange information represented? Such a result would provide evidence for the three-system account, supporting the idea that limits on infants' change detection are not necessarily the result of failures to form or maintain visual representations, but rather can be the result of how such representations are manipulated and used.

\section{Experiment 1}

Eleven-month-old infants participated in a two-phase procedure (Figure 1). In the introduction phase, the infants watched an event in which a short object became much taller after being briefly hidden under a cover. This change event was produced in the same way as that in Wang \& Baillargeon (2006; W\&B hereafter). In the test phase, the infants received a pairedcomparison task wherein they were shown four static displays consisting of either the pre- or post-change object that they had seen before and an object of novel height.

The paired-comparison paradigm was used to assess recognition memory for the pre- and postchange objects, similar to the two-alternative forced-choice questions in Mitroff et al. (2004a). Because the change event in the introduction phase was identical to that of W\&B, the participants were of the same age, and the experiment was conducted with the same apparatus and stimuli, we inferred that the infants would fail to notice the change in the current experiment as well. According to our hypothesis, the infants' failure to detect the change was because the physical-reasoning system did not use the information already stored in the objectrepresentation system. The paired-comparison task involved static displays with no object movement or interaction, and it asked not whether the infants could notice impossible outcomes of a physical event but whether they could recognize the previously seen objects. Because the task taps only the object-representation system, the infants in the present experiment should be able to access the information stored in that system, recognize the previously seen objects, and discriminate them from novel objects. Thus, we predicted a novelty-preference response: infants should look reliably longer at the novel than at the pre- or post-change object. ${ }^{3}$

\section{Method}

Participants-Sixteen healthy full-term infants participated ( 9 female; $M=11$ months 7 days; range: 10 months 14 days to 11 months 18 days). An additional five infants were tested but failed to complete any test trial owing to fussiness (3) or inattentiveness (2).

\footnotetext{
${ }^{3}$ When a novel item is paired with a previously seen item, whether it will elicit a novelty or familiarity preference is generally determined by infant age, stimulus complexity, and exposure duration. A novelty preference is more likely to be observed with older infants, simpler stimuli, and longer exposures (e.g. Colombo, Mitchell \& Horowitz, 1988; Hunter \& Ames, 1988). Novelty preferences have been observed in 6-month-old infants with an exposure to multiple objects for $7.5 \mathrm{~s}$ (Richards, 1997). Our 11-month-old infants were exposed to the pre- and post-change objects, each for $10 \mathrm{~s}$, during the change event (see Procedure); thus, we predicted a novelty-preference response. However, the current focus is whether or not infants can recognize the pre- and/or post-change objects and discriminate them from the novel object. Any systematic response pattern would be informative.
} 
Materials-The apparatus used during the introduction phase was the same as in W\&B. An experimenter produced the change event through a small window in the back wall of the apparatus. An object protruded through a hole in the apparatus floor and was attached to a platform under the floor. During the introduction phase, another experimenter lifted the platform to extend the visible portion of the object at the end of the event, changing the object from short to tall. Between the introduction and the test phase, the apparatus was modified in two ways. First, the back wall was replaced with a new wall containing a small hole, $2 \mathrm{~cm}$ in diameter, for video recording. Second, the floor was overlaid by a new surface with no hole.

The stimuli used during the introduction phase were identical to those in $\mathrm{W} \& \mathrm{~B}$, including a blue cover ( $11.5 \mathrm{~cm}$ in diameter, $16.5 \mathrm{~cm}$ tall) and a red object $(5.5 \mathrm{~cm}$ wide, $5.5 \mathrm{~cm}$ deep). The object height was $4.5 \mathrm{~cm}$ at the beginning and $15.5 \mathrm{~cm}$ at the end of the event. During the test phase, three objects ( $5.5 \mathrm{~cm}$ wide, $5.5 \mathrm{~cm}$ deep) were used: a pre-change $(4.5 \mathrm{~cm}$ tall $)$, a post-change $(15.5 \mathrm{~cm}$ tall) and a novel $(10 \mathrm{~cm}$ tall) object. The novel object was always paired with either the pre- or the post-change object; the two objects were placed $51 \mathrm{~cm}$ apart and 34 $\mathrm{cm}$ from the front of the apparatus.

Procedure-Each infant received a single introduction trial and four test trials. During the introduction phase, the infant saw the change event conducted as in W\&B. The infant first looked at a display consisting of the cover and short object for two cumulative seconds. Then, a gloved hand grasped the cover $(2 \mathrm{~s})$ and rotated it upward to show the hollow interior $(2 \mathrm{~s})$. After the infant had looked at this scene for two cumulative seconds, the hand lowered the cover to its starting location ( $2 \mathrm{~s})$, lifted it $(1 \mathrm{~s})$, moved it to the right directly above the object $(1 \mathrm{~s})$, and lowered it over the object ( $2 \mathrm{~s})$. After a 2-s pause, the hand grasped the cover again $(2 \mathrm{~s})$, lifted it ( $2 \mathrm{~s})$, moved it to the left (1 s), and returned it to the starting location ( $2 \mathrm{~s})$, revealing a much taller object. When the infant had looked at the final paused scene for 10 cumulative seconds, a cloth-covered frame was lowered to conceal the entire stimulus area, ending the introduction phase. Notice that from the beginning of the introduction phase, the initial portion of the event involving the pre-change object lasted $10 \mathrm{~s}$ before the cover was lifted; this design ensured that the infant was exposed to the pre- and post-change objects for the same duration.

After a short pause ${ }^{4}$ in which the apparatus was prepared for the test trials, the frame was raised to reveal the first of four static test displays. Each test display consisted of an object of novel height paired with either the pre- or post-change object. The novel (medium height) object appeared twice on the left and twice on the right, paired with either the pre-change (short height) or the post-change (tall height) object. The order of the test displays was counterbalanced across participants. Each test trial lasted $30 \mathrm{~s}$.

The infant sat on the parent's lap approximately $50 \mathrm{~cm}$ from and centred in front of the apparatus; the parent was instructed to remain silent and to close his or her eyes during each trial. Two observers monitored the infant's looking behaviour through peepholes in large clothcovered frames on either side of the apparatus. During the introduction phase, the primary (and typically more experienced) observer determined when the trial ended; inter-observer agreement for the introduction phase in this and the following experiment averaged $94 \%$ per infant. During the test trials, the computer terminated each trial after $30 \mathrm{~s}$. Two observers determined the infant's eye gaze online; each observer determined how long the infant looked at one of the two objects. An additional observer coded 30\% of the recorded responses offline; reliability between online and offline observations was $93 \%$.

\footnotetext{
${ }^{4}$ The delay between the introduction and test phases averaged $79.00 \mathrm{~s}$ in Experiment 1 and $85.81 \mathrm{~s}$ in Experiment 2.
} 
Replication-To strengthen our inference that the current participants would fail to notice the change event as in $\mathrm{W} \& \mathrm{~B}$, we tested 16 additional infants ( 7 male; $M=11$ months 7 days; range: 10 months 14 days to 11 months 18 days), using the procedure of the covering condition in W\&B. The infants who saw the change and those who saw the no-change event tended to look equally (change: $M=14.78 \mathrm{~s}$, no-change: $16.89 \mathrm{~s} ; t(15)<1$ ), replicating the previous findings that 11-month-olds fail to detect the change in covering events.

\section{Results and discussion}

Preliminary analyses revealed that all infants in this and the following experiment had looked at both objects in each test trial. In addition, no significant effects involving sex or order were obtained; thus, data were collapsed across sex and order in subsequent analyses.

The infants' averaged looking times at the pre-/post-change object were collapsed and compared with their averaged looking times at the novel object with a paired $t$-test (Figure 2). The analysis yielded a significant effect: the infants looked reliably longer at the novel $(M=$ $6.27 \mathrm{~s})$ than at the pre-/post-change $(M=5.20 \mathrm{~s})$ object $(t(15)=2.52, p<.025$, Cohen's $d=$. $65)$. However, different results were obtained when analysing the pre- and post-change object trials separately. The infants looked reliably longer at the novel $(M=6.27 \mathrm{~s})$ than at the postchange ( $M=4.84 \mathrm{~s})$ object $(t(15)=2.82, p<.025, d=.73)$, but did not look significantly longer at the novel $(M=6.28 \mathrm{~s})$ than at the pre-change $(M=5.56 \mathrm{~s})$ object $(t(15)<1)$.

Although there was a trend that the infants preferred the novel over the pre-change object, the difference in their looking times was not significant. There are two possible explanations for this result. It could be that the pre-change height was not maintained in memory. This explanation is in line with prior adult research showing that post-change information can overwrite or disrupt pre-change information (e.g. Beck \& Levin, 2003). Alternatively, it could be that the pre-change height was maintained, but in a somewhat fragile format. That is, the infants might have represented the pre-change height in the introduction phase, but this representation deteriorated over the course of the test phase. Indeed, Mitroff et al. (2004a) found that pre-change information was held in memory when observers failed to detect the change, but, importantly, it was represented in a weaker form than post-change information. It is possible that the mixed-test-trials design of Experiment 1 (i.e. where the pre- and postchange objects were alternately paired with the novel object in the test displays) may have further weakened the already fragile pre-change representations, resulting in the infants' failure to discriminate between the novel and the pre-change object. Experiment 2 tested this possibility.

\section{Experiment 2}

The infants in Experiment 2 watched an event identical to that in Experiment 1 during the introduction phase and then saw test displays consisting of the pre-change and novel objects; the post-change object was not used during the test phase. We reasoned that if the negative result in Experiment 1 was derived from the mixed-test-trials design, this modification might reduce the difficulty for infants to maintain their representation of the pre-change height throughout the test phase. As a result, they should now look reliably longer at the novel than at the pre-change object.

\section{Methods}

The design was identical to Experiment 1, except that during the test phase the infants saw four test displays with only the novel and pre-change objects (the object location was counterbalanced). Sixteen infants participated ( 7 female; $M=11$ months 5 days; range: 10 months 12 days to 11 months 23 days). Four of them became overly fussy after two test trials, 
terminating their participation; the following analyses included their responses with only two test trials.

\section{Results and discussion}

The infants' looking times at the novel and pre-change objects during the test trials (Figure 2) were averaged and compared as in Experiment 1 . The analysis indicated that the infants looked reliably longer at the novel $(M=6.25 \mathrm{~s})$ than at the pre-change $(M=4.66 \mathrm{~s})$ object $(t(15)=$ $3.99, p<.003, d=1.03)$. An additional analysis focusing only on the first two test trials also yielded a significant effect (novel: $M=6.97 \mathrm{~s}$; pre-change: $M=5.15 \mathrm{~s} ; t(15)=2.28, p<.05$, $d=.59$ ). Together with Experiment 1, these results support the claim that infants can form and maintain representations of the pre- and post-change heights of the object even when they fail to detect the change. ${ }^{5}$

\section{Additional results}

One could argue that the infants' longer looking times at the novel than at the pre- or postchange object may reflect an intrinsic bias for the particular height of the novel object $(10 \mathrm{~cm})$ or a transient preference derived from the repeated exposure to the novel object in every test trial. Although they are unlikely, we examined these possibilities by testing an additional 16 infants ( 7 female; $M=10$ months 28 days; range: 10 months 12 days to 11 months 20 days) with only the test phase, during which the pre- and post-change objects were alternately paired with the novel object as in Experiment 1. These infants did not watch the change event and had no prior exposure to the pre- or post-change object. We reasoned that if the positive results reflected an intrinsic preference over the 10-cm height or a transient bias derived from seeing the novel object in every test trial, the infants in this control condition should exhibit the same preference and look reliably longer at the novel than at the pre-/post-change objects. The results indicated that the infants looked about equally at the novel $(M=4.82 \mathrm{~s})$ and pre-/post-change $(M=5.27 \mathrm{~s})$ objects $(t(15)<1)$. The same pattern was obtained when analysing pre- and postchange object trials separately $(t s<1.29, p s>.10)$. Together, the results ruled out the alternative interpretations.

\section{General discussion}

The present experiments demonstrated that, although 11-month-old infants failed to notice a change to an object's height in a covering event, they had nevertheless encoded information about the pre- and post-change heights of the object. These representations were maintained in a useable format, allowing them to distinguish between the novel object and the pre- or postchange object during the test phase. Thus, just as were the adults in previous research (Mitroff et al., 2004a), infants are capable of simultaneously representing pre- and post-change information, and can suffer 'change blindness' from a failure to use available representations. The present finding sheds new light on the nature of visual representations in infancy and is consistent with prior demonstrations that infants' representational abilities do not always translate into successful task performance (e.g. Carey \& Xu, 2001; Xu \& Carey, 1996). For example, Xu \& Carey (1996) showed that 10-month-old infants noticed property differences between objects but failed to use this information to infer how many objects were present.

\footnotetext{
5 In Experiments 1 and 2, the infants watched the pre- and post-change objects during the introduction trial for the same duration; this design ensured that their test responses were not driven by unequal lengths of exposure to the pre- and post-change objects. Looking percentages were calculated by dividing the infants' looking time during the pre- or post-change portion of the event by the total amount of time taken to complete the portion. Both the pre- and post-change looking percentages were high, suggesting that the infants attended to the majority of each portion. However, the post-change looking percentage (86.89\%) was reliably lower than the pre-change percentage $(94.60 \%)(t(31)=2.50, p<.025)$, indicating that the infants gradually lost interest during the event. This result supports that the infants did not detect the change; had they noticed the change, they would have been intrigued by it and exhibited heightened interest in the postchange portion of the event.
} 
There remains no direct explanation for why adults may fail to use or compare available representations; even with an explanation for the adult data though, different mechanisms could underlie infants' responses. Although the current results mirror those of Mitroff $e t$ al. (2004a), the tasks varied greatly. The adults viewed whole-object changes, whereas the infants viewed a property change (i.e. a change in height in an otherwise identical object); the adults were explicitly told to look for a change, whereas the infants were not. Thus, it remains an open question whether the same mechanisms underlie the two studies. Here we consider one possible mechanistic explanation for the infant data.

According to the theoretical account described earlier (Wang \& Baillargeon, 2008), the physical-reasoning system categorizes a physical event, taps infants' knowledge about the event category, and queries the object-representation system for information about the variables that have been identified as relevant for the category. When infants have not identified a variable as relevant to an event category, information about this variable is not retrieved for the purpose of physical reasoning, even though it may still be available in another representational system. In the present experiments, the infants watched static displays in the test trials and were engaged in a recognition process to judge whether they had seen the objects before. This was a task that did not require the physical-reasoning system. As predicted by the account, the infants successfully used the information stored in the object-representation system to discriminate between the novel and the pre- or post-change object. To our knowledge, the present report is among the first to provide evidence for demarcation between the representational system of object recognition and that of physical reasoning, showing that although one system may use a particular piece of information (e.g. the height information of the red object used in the experiment), the other may not.

To conclude, our results indicate that when infants fail to detect a change, they nevertheless preserve more than one visual representation. Furthermore, they can form and maintain such multiple representations along the same physical dimension - here we show that infants simultaneously represent two unique object heights in visual memory. Thus, the present research continues the fruitful enterprise of comparing infant and adult object knowledge (e.g. Carey \& Xu, 2001; Cheries, Mitroff, Wynn \& Scholl, 2008; Leslie et al., 1998; Mitroff, Scholl \& Wynn, 2004; Scholl \& Leslie, 1999). It demonstrates robust representational abilities in infants, shows that infants behave similarly to adults in analogous scenarios, and provides a new approach for linking the investigations of infant cognition and adult perception.

\section{Acknowledgments}

This research was supported by a Junior Scholar Grant from the Chiang Ching-kuo Foundation and by a Special Research Grant from UCSC to SW. SRM was supported by NIMH \#R03 MH080849. We thank Renée Baillargeon, Andrea Frick, Jennifer Rigney and Dan Simons for helpful comments; Elena Hoicka, Philicia Rubin, Laura Trombetta and the undergraduate research assistants in the Infant Development Lab at UCSC for their help with data collection; and the parents and infants who kindly participated in the research.

\section{References}

Baillargeon R. Innate ideas revisited: for a principle of persistence in infants' physical reasoning. Perspectives on Psychological Science 2008;3:2-13.

Baillargeon R, DeVos J. Object permanence in young infants: further evidence. Child Development 1991;62:1227-1246. [PubMed: 1786712]

Baillargeon, R.; Li, J.; Ng, W.; Yuan, S. An account of infants' physical reasoning. In: Woodward, A.; Needham, A., editors. Learning and the infant mind. New York: Oxford University Press; in press

Beck MR, Levin DT. The role of representation volatility in recognizing pre- and post-change objects. Perception \& Psychophysics 2003;65:458-468. [PubMed: 12785074] 
Carey S, Xu F. Infants' knowledge of objects: beyond object files and object tracking. Cognition (Special issue: Objects and attention) 2001;80:179-213.

Casasola M. When less is more: How infants learn to form an abstract categorical representation of support. Child Development 2005;76:279-290. [PubMed: 15693772]

Cheries EW, Wynn K, Scholl BJ. Interrupting infants' persisting object representations: an object-based limit? Developmental Science 2006;9:F50-F58. [PubMed: 16911437]

Cheries EW, Mitroff SR, Wynn K, Scholl BJ. Cohesion as a constraint on object persistence in infancy. Developmental Science 2008;11:427-432. [PubMed: 18466376]

Colombo J, Mitchell DW, Horowitz FD. Infant visual attention in the paired-comparison paradigm: Testretest and attention-performance relations. Child Development 1988;59:1198-1210. [PubMed: 3168636]

Feigenson L, Carey S, Hauser M. The representations underlying infants' choice of more: object files versus analog magnitudes. Psychological Science 2002;13:150-156. [PubMed: 11933999]

Hespos SJ, Baillargeon R. Infants' knowledge about occlusion and containment events: a surprising discrepancy. Psychological Science 2001;12:140-147.

Hespos SJ, Baillargeon R. Décalage in infants' knowledge about occlusion and containment events: converging evidence from action tasks. Cognition 2006;99:B31-B41. [PubMed: 15939414]

Hollingworth A. Failures of retrieval and comparison constrain change detection in natural scenes. Journal of Experimental Psychology: Human Perception and Performance 2003;29:388-403. [PubMed: 12760623]

Hunter MA, Ames EW. A multifactor model for infant preferences for novel and familiar stimuli. Advances in Infancy Research 1988;5:69-95.

Káldy Z, Leslie A. A memory span of one? Object identification in 6.5-month-old infants. Cognition 2005;97:153-177. [PubMed: 16226561]

LeCompte GK, Gratch G. Violation of a rule as a method of diagnosing infants' level of object concept. Child Development 1972;43:385-396.

Leslie, AM. ToMM, ToBY, and agency: core architecture and domain specificity. In: Hirschfeld, LA.; Gelman, SA., editors. Mapping the mind: Domain specificity in cognition and culture. New York: Cambridge University Press; 1994. p. 119-148.

Leslie AM, Xu F, Tremoulet PD, Scholl BJ. Indexing and the object concept: developing 'what' and 'where' system. Trends in Cognitive Sciences 1998;2:10-18.

Luo Y, Baillargeon R. When the ordinary seems unexpected: evidence for incremental physical knowledge in young infants. Cognition 2005;95:297-328. [PubMed: 15788161]

Mitroff SR, Scholl BJ, Wynn K. Divide and conquer: how object files adapt when a persisting object splits into two. Psychological Science 2004a;15:420-425. [PubMed: 15147497]

Mitroff SR, Simons DJ, Levin DT. Nothing compares 2 views: change blindness can result from failures to compare retained information. Perception \& Psychophysics 2004b;66:1268-1281. [PubMed: 15813193]

Muller AA, Aslin RN. Visual tracking as an index of the object concept. Infant Behavior \& Development 1978;1:309-319.

Needham A. Object recognition and object segregation in 4.5-month-old infants. Journal of Experimental Child Psychology 2001;78:3-24. [PubMed: 11161419]

Newcombe N, Huttenlocher J, Learmonth A. Infants' coding of location in continuous space. Infant Behavior \& Development 1999;22:483-510.

O'Regan JK, Noë A. A sensorimotor account of vision and visual consciousness. Behavioral and Brain Sciences 2001;24:939-1031. [PubMed: 12239892]

Pashler H. Familiarity and visual change detection. Perception and Psychophysics 1988;44:369-378. [PubMed: 3226885]

Pylyshyn ZW. Visual indexes, preconceptual objects, and situated vision. Cognition 2001;80:127-158. [PubMed: 11245842]

Richards JE. Effects of attention on infants' preference for briefly exposed visual stimuli in the pairedcomparison recognition-memory paradigm. Developmental Psychology 1997;33:22-31. [PubMed: 9050387] 
Rigney, J.; Wang, S. Behind versus inside: infants form an abstract category of containment events. Poster accepted to be presented at the meeting of the Society for Research in Child Development; Denver, CO. 2009; 2009.

Scholl, BJ.; Leslie, AM. Explaining the infant's object concept: beyond the perception/cognition dichotomy. In: Lepore, E.; Pylyshyn, Z., editors. What is cognitive science?. Oxford: Blackwell; 1999. p. 26-73.

Simons DJ, Rensink RA. Change blindness: past, present, and future. Trends in Cognitive Sciences 2005;9:16-20. [PubMed: 15639436]

Spelke ES. Initial knowledge: six suggestions. Cognition 1994;50:431-445. [PubMed: 8039373]

Wang, S. What you believe is what you see: categorization makes the same event appear different to infants. Paper accepted to be presented at the symposium Selective use of information in infancy: the roles of categorization and action, Society for Research in Child Development; Denver, CO. 2009; 2009.

Wang S, Baillargeon R. Infants' physical knowledge affects their change detection. Developmental Science 2006;9:173-181. [PubMed: 16472318]

Wang S, Baillargeon R. Detecting impossible changes in infancy: a three-system account. Trends in Cognitive Sciences 2008;12:17-23. [PubMed: 18078778]

Wang S, Kohne L. Visual experience enhances 9-month-old infants' use of task-relevant information in an action task. Developmental Psychology 2007;43:1513-1522. [PubMed: 18020828]

Wang S, Baillargeon R, Paterson S. Detecting continuity violations in infancy: a new account and new evidence from covering and tube events. Cognition 2005;95:129-173. [PubMed: 15694644]

Wilcox T. Object individuation: infants' use of shape, size, pattern, and color. Cognition 1999;72:125166. [PubMed: 10553669]

Wilcox T, Chapa C. Infants' reasoning about opaque and transparent occluders in an individuation task. Cognition 2002;85:B1-B10. [PubMed: 12086715]

Xu F, Carey S. Infants' metaphysics: the case of numerical identity. Cognitive Psychology 1996;30:111153. [PubMed: 8635312] 
Introduction event

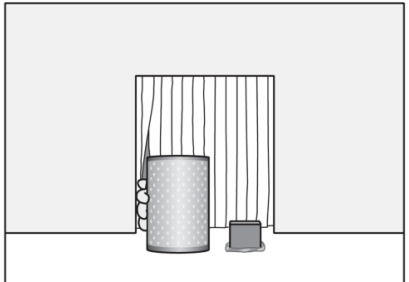

Test display 1

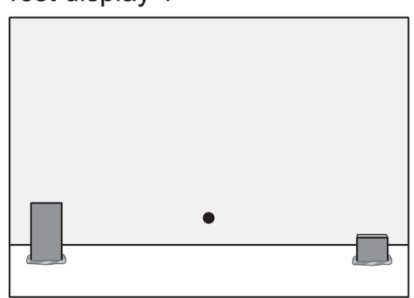

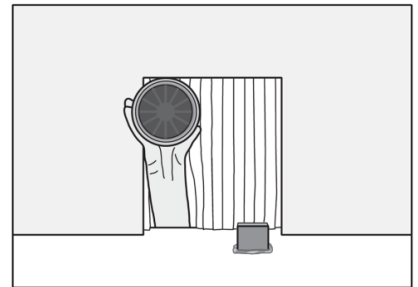

Test display 2

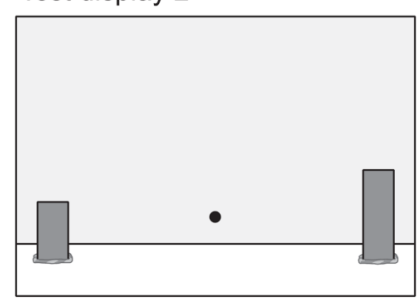

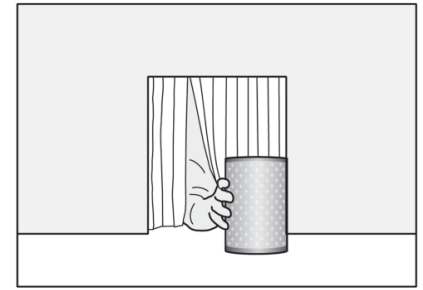

Test display 3

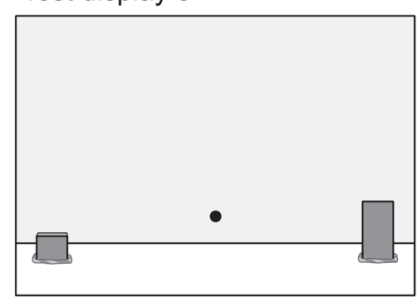

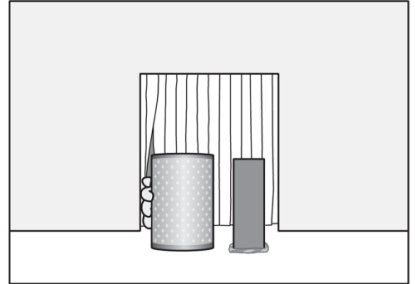

Test display 4

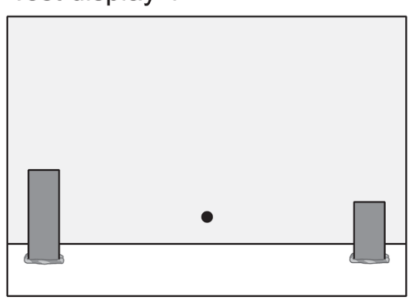

Figure 1.

Schematic drawings of (1) the sequential segments in the introduction event and (2) four possible test displays. 
Experiment 1
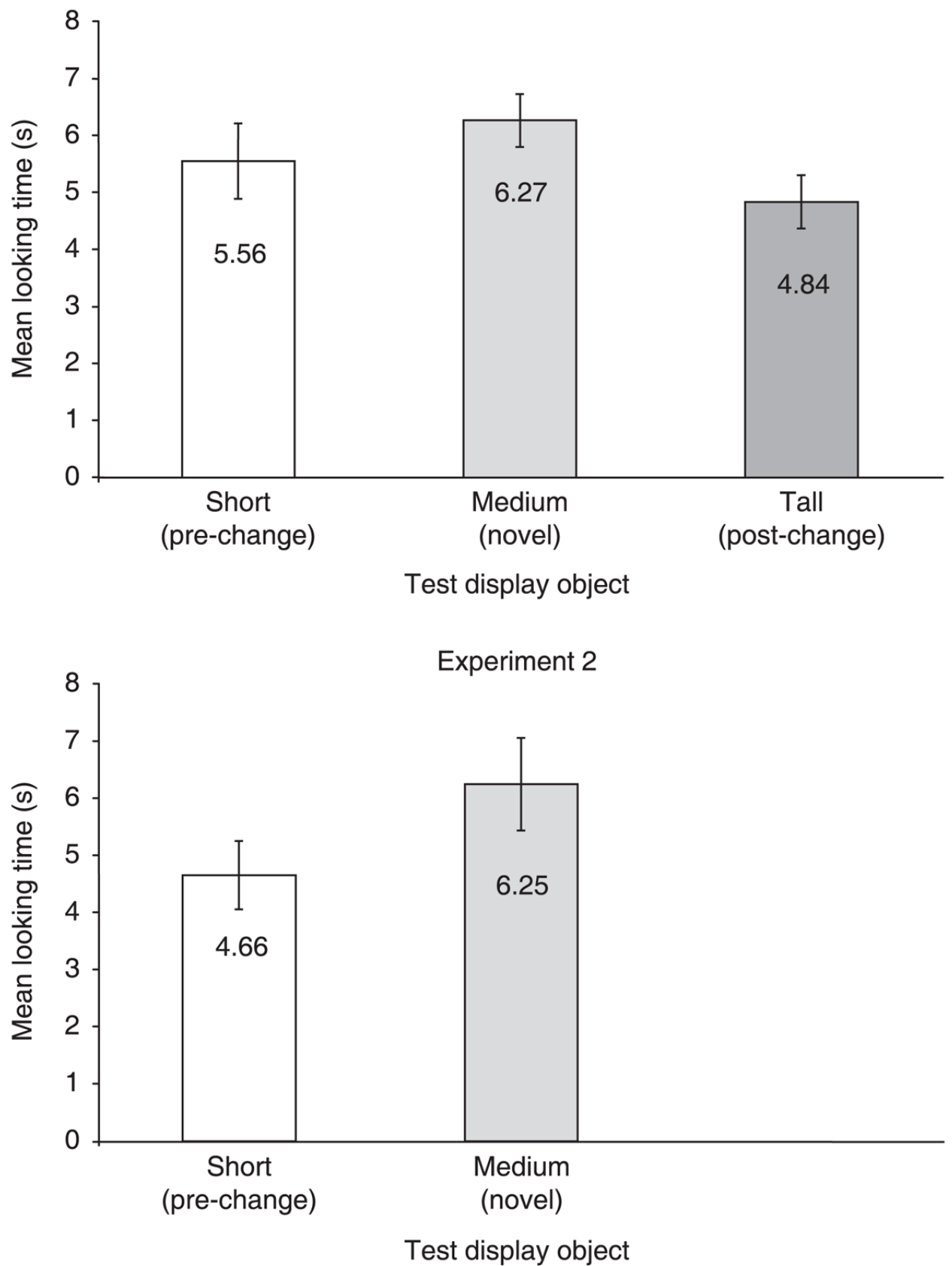

Figure 2.

Mean looking times of the infants in Experiments 1 and 2 at the pre-change, novel and postchange objects. Error bars represent $95 \%$ confidence intervals. 